\title{
Structures cohérentes et turbulence en écoulement libre
}

\section{Coherent structures and turbulence in shear layers}

\author{
M. Lesieur \\ Professeur à l'INPG \\ Institut de mécanique de Grenoble
}

On montre par simulation numérique directe des équations de Navier-Stokes bidimensionnelles que les tourbillons de Kelvin-Helmholtz en couche de mélange donnent naissance à un champ de turbulence bidimensionnelle. Celle-ci est caractérisée par son imprédicibilité, ainsi que par un spectre spatial d'énergie cinétique à bande large. Dans le cas du jet plan (jet de Bickley), on met en évidence l'apparition d'une allée de Karman, dont la régularité depend de la nature de la petite perturbation superposée à l'écoulement de base. On discute enfin de la tridimensionnalisation des rouleaux de Kelvin-Helmholtz.

It is shown, with the aid of direct numerical simulations of the two-dimensional Navier-Stokes equations, that the mixing-layer Kelvin-Helmholtz eddies evolve into a two-dimensional turbulence field. The latter is characterized both by its unpredictability and by the existence of a broad-band spatial kinetic energy spectrum. In the case of a plane jet (Bickley jet), one shows the appearance of a Karman street, whose regularity depends on the nature of the small perturbation superposed upon the basic flow. Finally, the three-dimensionalization of the Kelvin-Helmholtz vortices is discussed.

\section{Les structures cohérentes dans la nature}

L'existence de structures spatialement organisées" au sein des écoulements turbulents est une évidence reconnue depuis que l'homme a commencé à observer son environnement afin de le comprendre : comme l'a fort joliment commenté M. Serres [1], on pourrait peut-être attribuer à Lucrèce [2] le concept de structure cohérente. En effet ce dernier propose que le monde et toutes les structures vivantes résultent de l'apparition de structures organisées au sein d'un chaos Brownien originel. Plus près de nous, Van Gogh peint, dans «la nuit étoilée », des tourbillons d'étoiles s'appariant comme les tourbillons de KelvinHelmholtz qui apparaissent dans une couche de mélange entre deux écoulements de vitesse différente. Les cartes météorologiques nous montrent quotidiennement des structures cycloniques ou anticycloniques qui, bien que cohérentes, ont cependant une évolution impossible à prévoir au-delà de quelques jours. Nous dirons qu'un tel comportement est imprédicible. Des tourbillons analogues sont observés par les satellites dans l'océan, dans les grands courants tels que le Gulf stream ou le Kuroshio par exemple. Les sillages d'obstacles tels que automobiles, avions ou engins profilés sont, à grand nombre de Reynolds, de véritables allées tourbillonnaires, mais qui ont perdu le comportement reproductible et prédicible qu'elles avaient à plus bas nombre de Reynolds. On pourrait multiplier les exemples de structures cohérentes dans les écoulements de couche limite $[3]^{(2)}$, en rotation (écoulement de Couette-Taylor) ou en convection thermique. En fait les observations montrent qu'un écoulement

(1) C"est-à-dire ayant une forme particulière reconnaissable

(2) On les appelle alors tourbillons en épingle à cheveux. 
turbulent est en géneral composé à la fois de structures cohérentes $^{(3)}$ et de turbulence tridimensionnelle à plus petite échelle ayant souvent un caractère d'isotropie, et dont le spectre d'énergie cinétique suit la fameuse loi de Kolmogorov [4] en $k^{-53}$.

Le but du présent exposé est de développer le point de vue que les structures cohérentes ne sont pas des structures pathologiques qu'il faut exclure de la notion de turbulence, et dont il faut considérer la dynamique à part. $\mathrm{Au}$ contraire, les structures cohérentes sont une partie intégrante $^{(t)}$ de la turbulence, dont elles ont tous les caractères d'imprédicibilité et de diffusion.

Dans les écoulements libres, tels que couches de mélange, jets ou sillages par exemple, les structures cohérentes sont des tourbillons de type rouleaux de Kelvin-Helmholtz qui sont quasi bidimensionnels. Ces structures correspondent à la notion importante de turbulence bidimensionnelle (voir par exemple [5]), à laquelle est superposée la turbulence tridimensionnelle petite échelle dont il a été parlé ci-dessus.

\section{La turbulence bidimensionnelle en couche de mélange}

L'exemple le plus simple de structure cohérente est sans doute obtenu par instabilité de Kelvin-Helmholtz dans un écoulement présentant un profil de vitesse inflexionnel. La théorie de la stabilité linéaire permet de montrer que, pour un nombre de Reynolds donné, il existe toujours une gamme de nombres d'onde instables: une petite perturbation à l'un de ces nombres d'onde s'amplifiera exponentiellement. Si la perturbation possède un spectre large, c'est la structure correspondant au nombre d'onde le plus amplifié qui l'emportera sur toutes les autres (mode fondamental), donnant naissance à des tourbillons de Kelvin-Helmholtz dont la longueur d'onde $\lambda_{i}$ est proportionnelle à l'épaisseur $\delta_{i}$ du cisaillement initial. La théorie de la stabilité montre que, dès que le nombre de Reynolds $U \delta_{i} / \nu$ dépasse quelques dizaines ${ }^{i s i}$, l'instabilité devient inviscide, dans le sens qu'elle ne dépend plus de la viscosité. La longueur d'onde dans la direction $x$ de l'écoulement du mode fondamental est donnée par [6]

$$
\lambda_{s}=7 \delta_{i}
$$

Ces structures sont clairement mises en évidence expérimentalement [7]. D'un point de vue numérique, on peut les simuler, à des nombres de Reynolds de plusieurs milliers, par résolution des équations de Navier-Stokes sur des gros calculateurs vectoriels : à un profil de vitesse de base

$$
\bar{u}(y)=U \tanh 2 y / \delta_{i},
$$

on superpose une perturbation aléatoire (bruit blanc) de très faible amplitude. L'ordinateur montre alors la croissance du mode fondamental de longueur d'onde $\lambda_{w}$, comme prédit par la théorie de l'instabilité linéaire. La perturbation évolue alors vers une allée de tourbillons de Kelvin-Helmholtz qui ont la même longueur d'onde : la figure I montre le champ de tourbillon dans un tel calcul [8]. Ces tourbillons vont ensuite avoir des appariements successifs (voir la figure 2), responsables de l'épaississement de la couche de mélange.

Une des caractéristiques de ces tourbillons est leur imprédicibilité : des petites modifications dans les perturbations initiales peuvent conduire à des écarts ultérieurs importants dans les champs de tourbillon. Ceci est en particulier visible sur la figure 3, qui montre, à un même instant, quatre réalisations de l'écoulement ne différant que dans la phase (et non l'amplitude) du bruit blanc initial [9]. Cette propriété d'imprédicibilité est importante pour notre propos, puisqu'elle montre que ces structures cohérentes constituent en fait un champ de turbulence bidimensionnelle. On peut enfin montrer (voir [8], [9]), que le spectre d'énergie cinétique de ces structures (spectre spatial longitudinal) devient un spectre large de pente comprise entre $k^{-4}$ et $k^{-3}$ après le premier appariement. Bien entendu, et dans un écoulement réel, de la turbulence tridimensionnelle à petite échelle (de spectre en $k^{-3}$ ) se superposera à cette turbulence bidimensionnelle.

Ces simulations numériques permettent aussi de calculer l'évolution d'un champ de température passif (c'est-à-dire sans réaction sur le champ de vitesse). Il apparait que la température est en fait un bien meilleur indicateur des structures cohérentes que le champ de tourbillon.

\section{L'allée de Von Karman turbulente}

Cette turbulence bidimensionnelle se retrouve, avec des interactions entre structures qui peuvent être encore plus riches, dans des écoulements où le profil de vitesse moyen ou de base présente plusieurs points d'inflexion ${ }^{161}$. C'est en particulier le cas d'un jet ou de l'écoulement derrière un obstacle ${ }^{171}$ : le profil de vitesse présente alors deux points d'inflexion, chacun responsable du lâcher d'une allée de tourbillons de Kelvin-Helmholtz, avec changement du signe des tourbillons d'une allée sur l'autre. Ces allées s'organisent en opposition de phase dans la direction $x$, ce qui correspond au mode dominant (dit sinueux) d'instabilité. Cette organisation alternée des tourbillons de signe opposé résulte en une allée de Von Karman. C'est ce que nous avons vérifié dans le cas de l'écoulement appelé jet de Bickley [10] : si ce jet est forcé par une petite perturbation déterministe au mode fondamental sinueux, superposée à la perturbation aléatoire, l'allée de Von Karman peut être extrêmement stable. Dans le cas du jet libre, c'est-à-dire non forcé, l'allée de Von Karman devient turbulente et perd sa régularité. Elle conserve néanmoins ses structures cohérentes, mais qui sont devenues imprédicibles. On observe en particulier des appariements de tourbillons de même signe, et la formation de dipoles de tourbillons de signe opposé.

(3) pouvant être de nature différente et à des échelles différenes.

(4) et parfois prépondérante.

(5) $U$ est la demi-différence de vitesse.

(6) Il faut cependant que ceux-ci correspondent à des maximas du tourbillon, selon le critère de Fjortoft.

(7) Dans ce dernier cas en effet, la présence de robstacle est responsable d'un déficit de vitesse moyenne. 

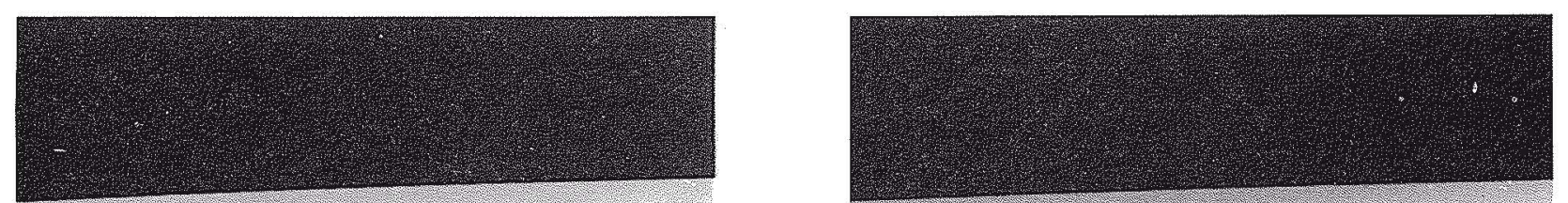

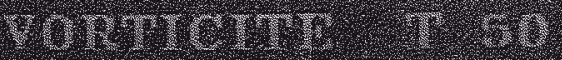

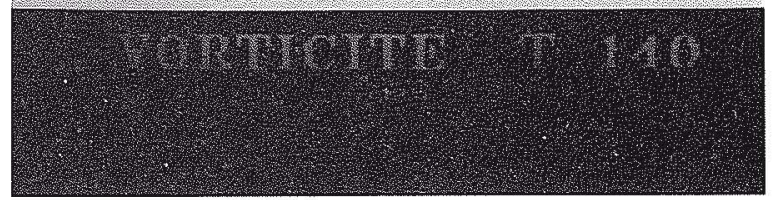

1. Toumillons de Kelvin-Helmholtz fondanentaux obtenus par simulation numérique (champ de tourbillon, d'après [8].

2. Appariemen de deux rourbillons de Kelvin-Helmholtz (dapres [S]).

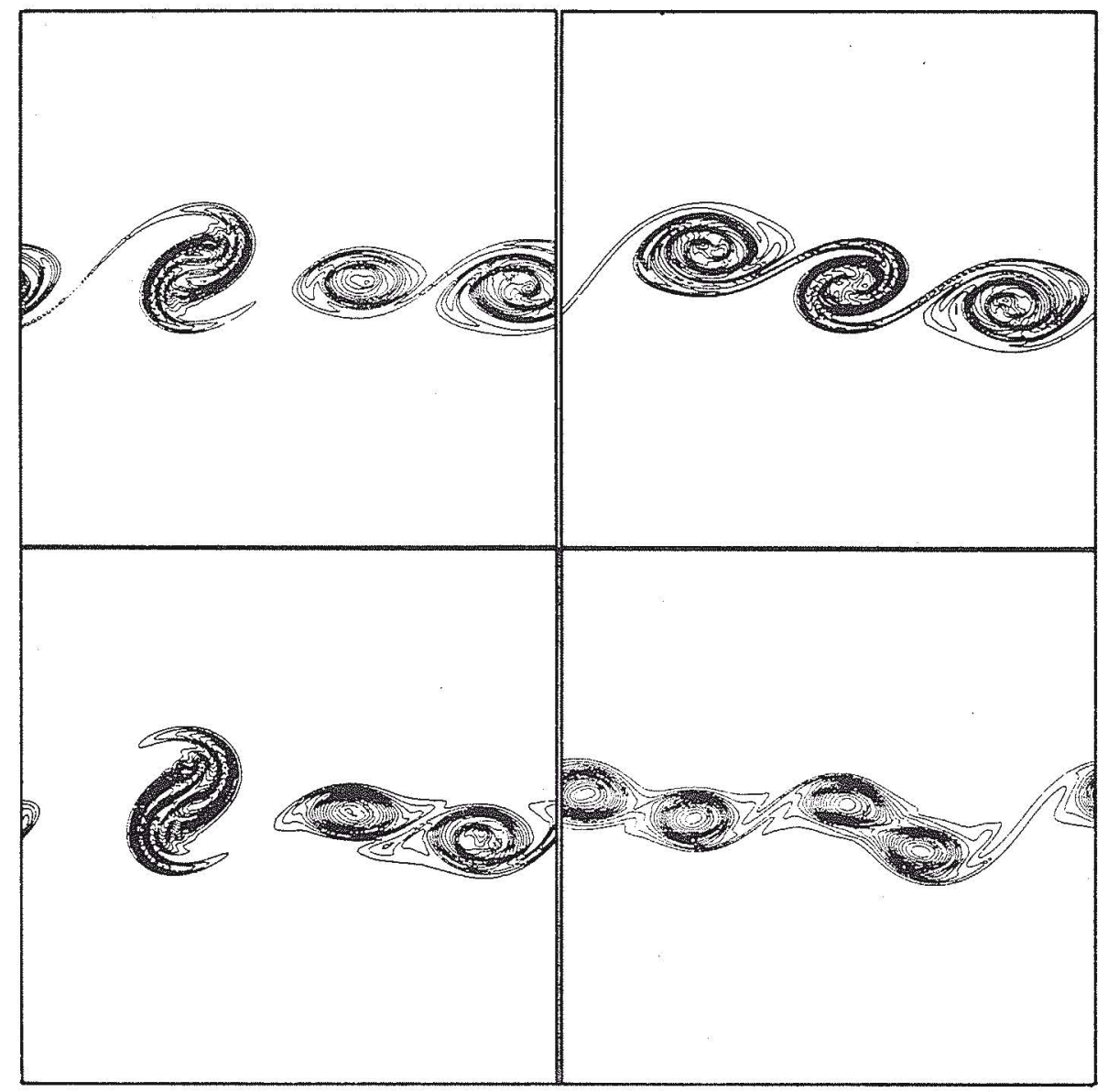

3. Champ de tourbillon apres plusieurs dizaines de temps de retournemen $\delta_{i} / U$ ), résultam d'un même érar de hase initial, mais différant par la perturbation superposée à l'état de base (d'après [5] et [9]). 


\section{La cohérence persiste-t'elle?}

Il semble donc maintenant bien compris comment, dans un cadre bidimensionnel, sont générées et évoluent les structures cohérentes. Dans la réalité cependant, ces structures ne sont plus contraintes par la bidimensionnalité : outre la turbulence tridimensionnelle à petite échelle déjà mentionnée, les rouleaux de Kelvin-Helmholtz sont soumis à des instabilités tridimensionnelles transverses (c'est-à-dire dans la direction de l'axe des rouleaux) qui sont encore assez mal comprises. On peut cependant faire ce que l'on appelle une troncature à deux modes de l'écoulement, en supposant qu'il existe une perturbation sinusoïdale ${ }^{(x)}$ dans cette direction. Un calcul analytique conduit alors à un système d'équations bidimensionnelles couplées pour le champ de base bidimensionnel et l'amplitude de la perturbation sinusoidale. La résolution numérique de ces équations [8] montre une croissance exponentielle de la perturbation tridimensionnelle. On peut donc envisager que certaines structures cohérentes vont se tridimensionnaliser par ce processus, et peut-être cascader par des instabilités successives vers de la turbulence tridimensionnelle. Dans ce sens, il est douteux que les structures cohérentes bidimensionnelles le restent indéfiniment. On peut néanmoins penser que, une fois la structure cohérente détruite par ce phénomène de croissance exponentielle de tridimensionnalité, l'instabilité linéaire du cisaillement de vitesse inflexionnel jouera de nouveau, avec pour résultat la recréation d'une nouvelle structure cohérente. L'évolution d'un écoulement turbulent libre aura donc un comportement cyclique de création et destruction continuelle de structures cohérentes.

\section{Les applications industrielles}

Ces méthodes de simulation numérique directe ou simulation des grandes échelles sont susceptibles de bouleverser profondément la philosophie de la modélisation des écoulements en situation industrielle. Elles nécessitent actuellement l'accès à de gros moyens de calcul scientifique, mais on peut raisonablement penser que des simulations bidimensionnelles comportant $128^{3}$ points de grille, ou tridimensionnelles à $32^{3}$ points de grille, vont se généraliser sur des mini-ordinateurs. Ces simulations permettront d'avoir accés à des quantités instantanées, telles que fluctuations de température par exemple, dont la détermination peut être cruciale dans le calcul de chambres de combustion, d'échangeurs thermiques ou de structures aérospatiales. Elles permettront aussi de donner une meilleure base physique à la modélisation statistique, que ce soit la modélisations en un point, ou, pour la turbulence homogène, la modélisation stochastique (cf. [5]).

(8) damplitude quelconque.

\section{Références}

[1] SeRres M.. 1977. - La naissance de la physique dans le texte de Lucrece, fleures et rurbulences. Editions de Minuit.

[2] LuCRECE. - "De natura rerum".

[3] MolN M. et Kıм J., 1982. - J. Fluid Mech., 118, pp. 341-378.

[4] Kolmogorov A.N., 1941. - Dokl. Akad. Nauk. SSSR, 30, pp. 301-305.

[5] Lesieur M., 1987. - Turbulence in Fhids. Nijhoff publishers.

[6] Michalke A., 1964. - J. Fluid Mech., 19. pp. 543-556.
[7] Brown G.L. et Roshko A., 1974 - J. Fluid Mech., 93 pp. 325-336.

[8] Lesieur M. Staquet C., Le Roy P. el Comte P., 1987 - The mixing laver and its coherence examined from the point of view of two-dimensional turbulence, I.M.G., preprint.

[9] Staquet C., Metals, et Lesieur M., 1985. - C.R. Acad. Sci. t 300 , ser. II, pp 833-838.

[10] COMTE P., Lesieur M. el Chollet J.P., 1987. - Simulation numérique du jet plan turbulen, l.M.G., preprint. 